\title{
Toxicogenomics in the Evolution of Toxicology
}

\section{Fang Liu* ${ }^{1 *}$ and Lei Guo*}

${ }^{1}$ Division of Neurotoxicology, National Center for Toxicological Research/Food and Drug Administration, AR, 72079, USA

${ }^{2}$ Biochemical Toxicology, National Center for Toxicological Research/Food and Drug Administration, AR, 72079, USA

\section{The Advent and Development of Toxicogenomics}

Advances in new molecular technologies have promoted the rapid development of toxicogenomics, which aims at understanding the response of the entire genome of an organism to toxicants/toxins. The primary goals of toxicogenomics are to understand the relationship between environmental stress and human disease susceptibility; to explore the molecular mechanisms of toxicants/toxins, and to identify biomarkers for predicting the onset of toxicity [1].

Toxicogenomics is defined as a combination of conventional toxicology with high-throughput technology. The term toxicogenomics was popularized in the literature in the late 1990s [2], when microarray technology emerged to display the capability of simultaneously monitoring the expression of thousands of genes. In this aspect, it is also referred as "transcriptomics". Toxicogenomics extended its scope to include metabonomics and proteomics when efficient detection methods for proteins and metabolites arose through high-throughput techniques. The use of the methods of "omics" offers opportunities of discovering biomarkers, revealing pathways, and learning how organisms respond to toxicant/toxin challenges. Since then, more than a decade of research has witnessed the evolution of toxicogenomics from isolated toxicant/toxin-induced gene-expression studies to full-scale studies with the integration of several "omics" strategies in toxicology [3-8].

\section{Transcriptomics in Toxicogenomic Study}

Microarray technology has proved to be a powerful tool in toxicological studies because it provides a platform for studying genome-wide gene expression patterns after toxicant/toxin exposure, which usually causes alterations of gene expression. It is generally believed that changes in gene expression are sensitive indicators of toxicity and thus are expected to be useful biomarkers of that toxicity. Moreover, gene expression profiles obtained from microarray analysis can improve our understanding the mechanisms of toxicity. For instance, Shi et al. [9] investigated the gene expression profile of ketamine (an $\mathrm{N}$-methyl-D-aspartate (NMDA) receptor antagonist)treated developing rat brain. They observed a significantly increased expression of the NMDA receptor in ketamine-treated brain. In addition, a series of studies from the same group investigated the neruotoxic effects of ketamine on developing brain. Higher expression of the NMDA receptor has been observed consistently and is closely related to the toxic effects of ketamine [10-12]. If ketamine's effect on the NMDA receptor during early development can be verified for other NMDA receptor antagonists, this common change in gene expression can indicate a unique mechanism by which NMDA receptor antagonists cause damage to the developing brain.

It is important to note that not all changes in gene expression can be explained as responses to a toxicant/toxin; altered gene expressions can result from homeostatic adjustments and other mechanisms in biological settings. Additionally, the use of different platforms and different analysis approaches also impact data interpretation. Thus, it is a challenge to identify accurately the real changes and minimize false positive and false negative results. The MicroArray Quality Control (MAQC) project, a community-wide effort led by the U.S. Food and Drug Administration, was established with the goals of assessing the performance of microarrays, searching for ways to minimize the poor concordance of gene expression data generated by various platforms and laboratories, and reaching consensus on methods of data analysis [13]. The findings from MAQC project demonstrated that reproducible measurement of differentially expressed genes across different platforms and laboratories for the same toxicants could be achieved, if microarray experiments were performed with strict quality control and adequate criteria are applied for data analysis [14].

\section{Proteomics and Metabonomics in Toxicogenomic Study}

The microarray is an efficient tool for examining the effects of toxicants/toxins at the transcription level; unfortunately, mRNA levels do not always parallel protein levels and protein function. Post-translational modifications of protein can change protein function, which is not necessarily reflected at the mRNA level. In addition, there are toxicants/toxins whose toxicities are due to their protein binding properties; genes may not be the direct targets. Thus, simple investigations of gene expression may miss certain important information.

The emergence of proteomics offers a way to analyze protein sequence, structure, and modifications, providing more functional understandings of toxic effects. It is viewed as a complementary approach to genomics, and understanding the relationship between mRNA and protein may guide the identification of more accurate, sensitive, and specific biomarkers $[8,15]$.

Alterations of mRNA and protein will result in changes of metabolite levels, but neither the genomic nor the proteomic approach can indicate what those changes are. Metabonomics, combining the application of analytical technologies, such as Nuclear Magnetic Resonance (NMR), with statistics, quantitatively measures the changes of endogenous metabolites in tissues and biofluids. Metabolites are the endpoints of the response an organism makes to a stimulus. Metabonomic measurements directly reflect the metabolism and physiology of an organism after toxicant/toxin exposure [16]. This is a promising and feasible method since body fluids, such as urine,

*Corresponding authors: Fang Liu, Division of Neurotoxicology, National Center for Toxicological Research/Food and Drug Administration, AR, 72079, USA, E-mail: Fang.Liu@fda.hhs.gov

Lei Guo, Biochemical Toxicology, National Center for Toxicological Research/ Food and Drug Administration, AR, 72079, USA, E-mail: Lei.Guo@fda.hhs.gov

Received July 30, 2012; Accepted August 24, 2012; Published August 26, 2012

Citation: Liu F, Guo L (2012) Toxicogenomics in the Evolution of Toxicology. J Pharmacogenom Pharmacoproteomics 3: e123. doi:10.4172/2153-0645.1000e123

Copyright: @ 2012 Liu F, et al. This is an open-access article distributed under the terms of the Creative Commons Attribution License, which permits unrestricted use, distribution, and reproduction in any medium, provided the original author and source are credited. 
blood, or cerebrospinal fluids are more readily available from human subjects. Furthermore, the continuous availability of these samples during toxicant/toxin exposure or during toxicant/toxin elimination makes it possible to search for biomarkers under a variety of treatment conditions [17].

Genomics, proteomics, and metabonomics have shown the ability to indentify biomarkers and provide information for toxic mechanism elucidation at the molecular level. It is anticipated that the integration of these "omics" methods will work in concert, or even synergistically, to provide a better understanding of toxic responses. More in-depth integration will rely on the development of toxicogenomics data repositories and more advanced technologies, such as next-generation sequencing.

It is worth mentioning that it is critical for scientists to choose carefully an appropriate platform and perform such high-throughput experiments with strict quality control. Alternative methods should be always considered to validate the results generated from "omics" before drawing conclusions. For example, changes of RNA or microRNA expression detected by a high-throughput technology should be validated using different gene expression platforms such as "TaqMan". Finally, although "omics" approaches for studying alterations in mRNA, proteins, and metabolites are critical in identifying molecular signatures, traditional methods, such as molecular biological, biochemical, and histological methods, are still indispensible for studying and verifying the changes of the molecules.

\section{Disclaimer}

This document has been reviewed in accordance with United States Food and Drug Administration (FDA) policy and approved for publication. Approval does not signify that the contents necessarily reflect the position or opinions of the FDA The findings and conclusions in this report are those of the authors and do not necessarily represent the views of the FDA.

\section{References}

1. Waters MD, Fostel JM (2004) Toxicogenomics and systems toxicology: aims and prospects. Nat Rev Genet 5: 936-948.

2. Nuwaysir EF, Bittner M, Trent J, Barrett JC, Afshari CA (1999) Microarrays and toxicology: the advent of toxicogenomics. Mol Carcinog 24: 153-159.

3. Coen M, Ruepp SU, Lindon JC, Nicholson JK, Pognan F, et al. (2004) Integrated application of transcriptomics and metabonomics yields new insight into the toxicity due to paracetamol in the mouse. J Pharm Biomed Anal 35: 93-105.
4. Ruepp SU, Tonge RP, Shaw J, Wallis N, Pognan F (2002) Genomics and proteomics analysis of acetaminophen toxicity in mouse liver. Toxicol Sci 65 : $135-150$.

5. lida M, Anna $\mathrm{CH}$, Hartis J, Bruno M, Wetmore B, et al. (2003) Changes in global gene and protein expression during early mouse liver carcinogenesis induced by non-genotoxic model carcinogens oxazepam and Wyeth-14,643. Carcinogenesis 24: 757-770.

6. Hogstrand C, Balesaria S, Glover CN (2002) Application of genomics and proteomics for study of the integrated response to zinc exposure in a nonmodel fish species, the rainbow trout. Comp Biochem Physiol B Biochem Mol Biol 133: 523-535.

7. Juan HF, Lin JY, Chang WH, Wu CY, Pan TL, et al. (2002) Biomic study of human myeloid leukemia cells differentiation to macrophages using DNA array, proteomic, and bioinformatic analytical methods. Electrophoresis 23: 24902504.

8. Heijne WH, Stierum RH, Slijper M, van Bladeren PJ, van Ommen B (2003) Toxicogenomics of bromobenzene hepatotoxicity: a combined transcriptomics and proteomics approach. Biochem Pharmacol 65: 857-875.

9. Shi Q, Guo L, Patterson TA, Dial S, Li Q, et al. (2010) Gene expression profiling in the developing rat brain exposed to ketamine. Neuroscience 166: 852-863.

10. Wang C, Sadovova N, Fu X, Schmued L, Scallet A, et al. (2005) The role of the $\mathrm{N}$-methyl-D-aspartate receptor in ketamine-induced apoptosis in rat forebrain culture. Neuroscience 132: 967-977.

11. Wang C, Sadovova N, Hotchkiss C, Fu X, Scallet AC, et al. (2006) Blockade of $\mathrm{N}$-methyl-D-aspartate receptors by ketamine produces loss of postnatal day 3 monkey frontal cortical neurons in culture. Toxicol Sci 91: 192-201.

12. Wang C, Sadovova N, Patterson TA, Zou X, Fu X, et al. (2008) Protective effects of 7-nitroindazole on ketamine-induced neurotoxicity in rat forebrain culture. Neurotoxicology 29: 613-620.

13. MAQC Consortium, Shi L, Reid LH, Jones WD, Shippy R, et al. (2006) The MicroArray Quality Control (MAQC) project shows inter- and intraplatform reproducibility of gene expression measurements. Nat Biotechnol 24: 11511161.

14. Guo L, Lobenhofer EK, Wang C, Shippy R, Harris SC, et al. (2006) Rat toxicogenomic study reveals analytical consistency across microarray platforms. Nat Biotechnol 24: 1162-1169.

15. Stierum R, Heijne W, Kienhuis A, van Ommen B, Groten J (2005) Toxicogenomics concepts and applications to study hepatic effects of food additives and chemicals. Toxicol Appl Pharmacol 207: 179-188.

16. Gatzidou ET, Zira AN, Theocharis SE (2007) Toxicogenomics: a pivotal piece in the puzzle of toxicological research. J Appl Toxicol 27: 302-309.

17. Lindon JC, Holmes E, Bollard ME, Stanley EG, Nicholson JK (2004) Metabonomics technologies and their applications in physiological monitoring, drug safety assessment and disease diagnosis. Biomarkers 9: 1-31. 